\title{
UNIVERSAL COLLECTIVE ROTATION CHANNELS AND QUANTUM ERROR CORRECTION
}

\author{
MARIUS JUNGE ${ }^{1}$, PETER T. KIM ${ }^{2}$, DAVID W. KRIBS ${ }^{2,3,4}$ \\ ABSTRACT. We present and investigate a new class of quantum \\ channels, what we call 'universal collective rotation channels', that \\ includes the class of collective rotation channels as a special case. \\ The fixed point set and noise commutant coincide for a channel \\ in this class. Computing the precise structure of this $\mathrm{C}^{*}$-algebra \\ is a core problem in a particular noiseless subsystem method of \\ quantum error correction. We prove that there is an abundance \\ of noiseless subsystems for every channel in this class and that the \\ Young tableaux combinatorial machine may be used to explicitly \\ compute these subsystems.
}

\section{INTRODUCTION}

The study of quantum channels is a central theme in quantum computing and quantum information theory [31]. A fundamental class of quantum channels is known as the class of collective rotation channels $[4,5,6,12,13,16,17,20,22,36,37,38,39,41]$. This class has its roots in the postulates of quantum mechanics and has recently played a key role in experimental efforts towards realizing certain quantum error correction methods [13, 37]. Of particular interest in the current study is the noise commutant method of noiseless subsystems. This is a recently developed paradigm for passive quantum error correction [11, 13, 17, 21, 22, 30, 40]. In this method, the 'noise commutant' is used as a vehicle for encoding states that are left immune to the noise of a given channel. The operator algebras generated by such states are called 'noiseless subsystems'.

In this paper, we present a new class of quantum channels and investigate them in the context of quantum error correction, with specific 
reference to the noiseless subsystem method. This class is a generalization of the collective rotation class, which arises as an important special case, hence we use the appellation 'universal collective rotation channels' to describe this class. We prove that the noise commutant for every channel in this class has rich structure and hence contains an abundance of noiseless subsystems. To accomplish this, we use operator algebra techniques to make an explicit connection with representation theory of the symmetric group and, as a consequence, the Young tableaux combinatorial machine [14, 18] may be used to explicitly compute these noiseless subsystems.

The paper is organized as follows. Section 2 contains introductory material on quantum channels and quantum error correction. In Section 3 we define and establish basic properties of the class of universal collective rotation (ucr-) channels. We make the connection with representation theory of the symmetric group in Section 4 and show that the noise commutant for ucr-channels is determined by a particular representation of the symmetric group. In Sections 5 and 6 we collect well-known facts from representation theory of the symmetric group, with emphasis on Young tableaux combinatorics, and work through some low-dimensional examples. We finish with a concluding remark in Section 7 and discuss possible avenues of further research.

\section{Quantum Channels and Noiseless Subsystems}

Let $\mathcal{H}$ be a (complex) Hilbert space and let $\mathcal{B}(\mathcal{H})$ be the set of bounded operators on $\mathcal{H}$. When a basis for $\mathcal{H}$ is fixed and $\operatorname{dim} \mathcal{H}=$ $k<\infty$, the algebra $\mathcal{B}(\mathcal{H})$ may be identified with the set of all complex $k \times k$ matrices $\mathbb{M}_{k}=\mathbb{M}_{k}(\mathbb{C})$. Throughout the paper, if we are given positive integers $n \geq 1$ and $d \geq 2$, we let $\{|0\rangle,|1\rangle, \ldots,|d-1\rangle\}$ be a

fixed orthonormal basis for $d$-dimensional Hilbert space $\mathcal{H}_{d}=\mathbb{C}^{d}$ and 
let $\left\{\left|i_{1} i_{2} \cdots i_{n}\right\rangle: i_{j} \in \mathbb{Z}_{d}\right\}$ be the corresponding orthonormal basis for $\mathcal{H}_{d^{n}}=\left(\mathbb{C}^{d}\right)^{\otimes n}$.

A linear map $\mathcal{E}: \mathcal{B}(\mathcal{H}) \rightarrow \mathcal{B}(\mathcal{H})$ is completely positive if for all $k \geq 1$ the ampliation maps $\mathbb{1}_{k} \otimes \mathcal{E}: \mathbb{M}_{k} \otimes \mathcal{B}(\mathcal{H}) \rightarrow \mathbb{M}_{k} \otimes \mathcal{B}(\mathcal{H})$ are positive. See [25, 32] for introductions to the study of completely positive maps from different perspectives. A quantum channel is a map $\mathcal{E}: \mathcal{B}(\mathcal{H}) \rightarrow \mathcal{B}(\mathcal{H})$ that is completely positive and trace preserving. Given $\mathcal{E}$, there is $\left([\mathbf{8},[26])\right.$ a set of noise operators, or errors, $\left\{E_{k}\right\}$ on $\mathcal{H}$ such that

$$
\mathcal{E}(\rho)=\sum_{k} E_{k} \rho E_{k}^{\dagger} \quad \text { for } \quad \rho \in \mathcal{B}(\mathcal{H}) .
$$

Trace preservation means that the noise operators satisfy

$$
\sum_{k} E_{k}^{\dagger} E_{k}=\mathbb{1}
$$

where $\mathbb{1}$ is the identity operator on $\mathcal{H}$. The channel is unital if also,

$$
\mathcal{E}(\mathbb{1})=\sum_{k} E_{k} E_{k}^{\dagger}=\mathbb{1} .
$$

We will denote the fixed point set for $\mathcal{E}$ by

$$
\operatorname{Fix}(\mathcal{E})=\{\rho \in \mathcal{B}(\mathcal{H}): \mathcal{E}(\rho)=\rho\} .
$$

Further let $\mathcal{A}$ be the algebra generated by $\left\{E_{k}\right\}$ from (11). This is called the interaction algebra in quantum computing [22]. It is a relic of the channel in the sense that the same algebra is obtained whatever the choice of noise operators in (11). This is most succinctly seen in the case of a unital channel. In general, $\operatorname{Fix}(\mathcal{E})$ is just a $\nmid$-closed subspace of $\mathcal{B}(\mathcal{H})$, but in the case of a unital channel $\mathcal{E}$, the so-called noise commutant

$$
\mathcal{A}^{\prime}=\left\{\rho \in \mathcal{B}(\mathcal{H}): \rho E_{k}=E_{k} \rho, \forall k\right\}
$$

coincides with the fixed point set [7, 28]:

$$
\operatorname{Fix}(\mathcal{E})=\mathcal{A}^{\prime}
$$


In particular, $\operatorname{Fix}(\mathcal{E})=\mathcal{A}^{\prime}$ is a $\dagger$-closed operator algebra (a finite dimensional $\mathrm{C}^{*}$-algebra $\left.[\mathbf{2}, \mathbf{1 0}, \mathbf{3 5}]\right)$. In this case the von Neumann double commutant theorem from operator algebras shows how the algebra $\mathcal{A}=\mathcal{A}^{\prime \prime}=\operatorname{Fix}(\mathcal{E})^{\prime}$ only depends on the channel.

Every finite dimensional $\mathrm{C}^{*}$-algebra is unitarily equivalent to an orthogonal direct sum of 'ampliated' full matrix algebras; i.e., there is a unitary operator $U$ such that

$$
U \mathcal{A} U^{\dagger}=\bigoplus_{k=1}^{r}\left(\mathbb{1}_{m_{k}} \otimes \mathbb{M}_{n_{k}}\right)
$$

From the representation theory perspective, a factor $\mathbb{1}_{m_{k}} \otimes \mathbb{M}_{n_{k}}$ corresponds to an $n_{k}$-dimensional irreducible representation appearing with multiplicity $m_{k}$. With this form for $\mathcal{A}$ given, the structure of the commutant up to unitary equivalence is easily computed by

$$
U \operatorname{Fix}(\mathcal{E}) U^{\dagger}=U \mathcal{A}^{\prime} U^{\dagger}=\bigoplus_{k=1}^{r}\left(\mathbb{M}_{m_{k}} \otimes \mathbb{1}_{n_{k}}\right) .
$$

(See [13, 17, 20, 36, 38, 39] for more detailed discussions in connection with quantum information theory.)

Given a quantum channel $\mathcal{E}$ with noise operators $\left\{E_{k}\right\}$, the structure of the noise commutant $\mathcal{A}^{\prime}$ can be used to prepare density operators for use in the noiseless subsystem method of error correction. This is a passive method of quantum error correction, in the sense that such operators will remain immune to the effects of the noise of the channel, without active intervention. Thus, computing the precise structure of $\mathcal{A}^{\prime}$ as in (2) is of fundamental importance in this method. We mention that for experimental reasons [29], only one matrix algebra $\mathbb{M}_{m_{k}} \otimes \mathbb{1}_{n_{k}}$ may be used at a time in this manner. Hence it is also desirable to find the largest full matrix algebra which is a subalgebra of the noise commutant. 


\section{Universal Collective Rotation Channels}

For the rest of the paper, given a positive integer $d \geq 2$ we write $\mathbb{M}_{d}$ for the operator algebra $\mathcal{B}\left(\mathbb{C}^{d}\right)$ represented as $d \times d$ complex matrices with respect to the standard basis $\{|0\rangle, \ldots,|d-1\rangle\}$ for $\mathbb{C}^{d}$. Further let $\mathbb{M}_{d, s a}$ be the subset of self-adjoint matrices inside $\mathbb{M}_{d}$.

Fix $n \geq 1$. Given $1 \leq k \leq n$ we define a representation of $\mathbb{M}_{d}$ on $\mathcal{H}_{d^{n}}$ by

$$
\omega_{k}(x)=\mathbb{1}_{d} \otimes \cdots \otimes \mathbb{1}_{d} \otimes \underbrace{x}_{k \text {-th position }} \otimes \mathbb{1}_{d} \otimes \cdots \otimes \mathbb{1}_{d}
$$

for all $x \in \mathbb{M}_{d}$. Then we may define sums of independent copies of $x$ by

$$
u_{n}(x)=\sum_{k=1}^{n} \omega_{k}(x) \quad \text { for } \quad x \in \mathbb{M}_{d}
$$

Definition 3.1. Given a finite subset $\mathcal{S} \subset \mathbb{M}_{d, s a}$, we define a universal collective rotation (ucr-) channel $\mathcal{E}_{\mathcal{S}}$ by

$$
\mathcal{E}_{\mathcal{S}}(\rho)=\frac{1}{\sqrt{|\mathcal{S}|}} \sum_{x \in \mathcal{S}} e^{i \theta_{x} u_{n}(x)} \rho e^{-i \theta_{x} u_{n}(x)} \quad \text { for } \quad \rho \in \mathcal{B}\left(\mathcal{H}_{d^{n}}\right),
$$

where $\left\{\theta_{x}: x \in \mathcal{S}\right\}$ are non-zero angles.

Given a set of operators $\mathcal{R}$, define $\operatorname{Alg} \mathcal{R}$ to be the operator algebra generated by $\mathcal{R}$. This is the set of all polynomials in the elements of $\mathcal{R}$. When $\mathcal{R}$ is a self-adjoint set, $\operatorname{Alg} \mathcal{R}$ is a $\mathrm{C}^{*}$-algebra. Through a standard functional calculus argument from operator theory, it follows that the interaction algebra $\mathcal{A}_{\mathcal{S}}$ for $\mathcal{E}_{\mathcal{S}}$ is obtained as $\mathcal{A}_{\mathcal{S}} \equiv \operatorname{Alg}\left\{e^{i \theta_{x} u_{n}(x)}: x \in\right.$ $\mathcal{S}\}=\operatorname{Alg}\left\{u_{n}(x): x \in \mathcal{S}\right\}$. Thus by von Neumann's double-commutant identity we have

$$
\mathcal{A}_{\mathcal{S}}=\left\{e^{i \theta_{x} u_{n}(x)}: x \in \mathcal{S}\right\}^{\prime \prime}=\left\{u_{n}(x): x \in \mathcal{S}\right\}^{\prime \prime} .
$$

Notice that $\mathcal{A}_{\mathcal{S}}$ is independent of the choice of (non-zero) angles $\theta_{x}$. As an application of the fixed point theorem from [7, 28] we obtain the following. 
Theorem 3.2. If $\mathcal{S}$ is a finite subset of $\mathbb{M}_{d, s a}$, then the ucr-channel $\mathcal{E}_{\mathcal{S}}$ satisfies

$$
\operatorname{Fix}\left(\mathcal{E}_{\mathcal{S}}\right)=\mathcal{A}_{\mathcal{S}}^{\prime}
$$

Observe that $\mathbb{1}_{d}$ belongs to $\mathcal{A}_{\mathcal{S}}$ from its characterization as a bicommutant. Since $u_{n}\left(\mathbb{1}_{d}\right)=n \mathbb{1}_{d^{n}}$, we may always add $\mathbb{1}_{d}$ to $\mathcal{S}$ without changing the properties of $\operatorname{Fix}\left(\mathcal{E}_{S}\right)$. This motivates the following definition.

Definition 3.3. We will say that $\mathcal{S}$ is maximal if $\operatorname{span}\{x: x \in \mathcal{S}\}$ contains all matrices $x \in \mathbb{M}_{d}$ with $\operatorname{tr}(x)=0$.

It turns out that for maximal $\mathcal{S}$ the algebra $\mathcal{A}_{\mathcal{S}}$ is a well-known object in representation theory.

Remark 3.4. To place the class of ucr-channels in context, we note that the ucr-channels for $d=2$ and general $n$ are the class of 'two-level' collective rotation channels from quantum computing [4, 5, $\mathbf{6}, \mathbf{1 2}, \mathbf{1 3}$, 16, 17, 20, 22, 36, 37, 38, 39, 41]. The noise operators in this case are also denoted by $J_{x}, J_{y}, J_{z}$ and they arise in quantum mechanics as the canonical representation of the angular momentum relations [9]. From the noiseless subsystem/quantum error correction perspective, the algebra $\operatorname{Fix}\left(\mathcal{E}_{\mathcal{S}}\right)=\mathcal{A}_{\mathcal{S}}^{\prime}$ for this subclass of ucr-channels, and natural $d$-dimensional representations of the $J_{k}$ operators, has been analyzed in [16] from an operator theory cum quantum mechanics point of view.

\section{Representation Theory and the Noise Commutant}

In this section we identify the structure of the noise commutant in terms of representation theory for the symmetric group. We begin with some notation. We shall denote the $n$-fold tensor product of $\mathbb{M}_{d}$ by

$$
\mathbb{M}_{d}^{\otimes n}=\underbrace{\mathbb{M}_{d} \otimes \cdots \otimes \mathbb{M}_{d}}_{n \text {-times }} \cong \mathbb{M}_{d^{n}} .
$$


Let $\operatorname{Sym}^{n} \mathbb{M}_{d}$ be the subalgebra of $\mathbb{M}_{d^{n}}$ generated by the symmetric tensor products; that is, $\operatorname{Sym}^{n} \mathbb{M}_{d}$ is the algebra generated by the operators

$$
\Phi_{n}\left(x_{1} \otimes \cdots \otimes x_{n}\right)=\frac{1}{n !} \sum_{\pi \in S_{n}} x_{\pi(1)} \otimes \cdots \otimes x_{\pi(n)},
$$

where each $x_{i} \in \mathbb{M}_{d}$ and $S_{n}$ is the permutation group on $n$ letters.

In terms of representation theory, we may equally well consider the representation $\pi: \mathrm{GL}(d) \rightarrow \mathrm{GL}\left(d^{n}\right)$ given by $\pi(u)=u \otimes \cdots \otimes u$, and then we have

$$
\operatorname{Sym}^{n} \mathbb{M}_{d}=\pi(\operatorname{GL}(d))^{\prime \prime},
$$

where GL $(d)$ is the group of $d \times d$ nonsingular complex matrices. This tensor product representation of GL $(d)$ is in 'duality' with the representation of the symmetric group $S_{n}$ defined on vector tensors by

$$
\pi(\sigma)\left(h_{1} \otimes \cdots \otimes h_{n}\right)=h_{\sigma(1)} \otimes \cdots \otimes h_{\sigma(n)},
$$

for $\sigma \in S_{n}$ and $h_{1}, \ldots, h_{n} \in \mathcal{H}_{d}$. In this context, Schur's classical duality theorem reads as

Theorem 4.1. $\pi\left(S_{n}\right)^{\prime}=\operatorname{Sym}^{n} \mathbb{M}_{d}$.

We use the following characterization of $\operatorname{Sym}^{n} \mathbb{M}_{d}$ below.

Lemma 4.2. For positive integers $d$ and $n$, we have

$$
\operatorname{Sym}^{n} \mathbb{M}_{d}=\left\{x^{\otimes n}: x \in \mathbb{M}_{d}\right\}^{\prime \prime}=\left\{u_{n}(x): x \in \mathbb{M}_{d}\right\}^{\prime \prime}
$$

Proof. It is clear that $\operatorname{Sym}^{n} \mathbb{M}_{d}$ contains the $\mathrm{C}^{*}$-algebra

$$
\begin{aligned}
\mathcal{B}=\left\{x^{\otimes n}: x \in \mathbb{M}_{d}\right\}^{\prime \prime} & =\operatorname{span}\left\{x^{\otimes n}: x \in \mathbb{M}_{d}\right\} \\
& =\operatorname{Alg}\left\{x^{\otimes n}: x \in \mathbb{M}_{d}\right\}
\end{aligned}
$$


as a subalgebra. For the converse inclusion, let $x_{1}, \ldots, x_{n} \in \mathbb{M}_{d}$ and consider the complex matrix integral

$$
\begin{aligned}
& \int_{z_{1}, \ldots, z_{n} \in \mathbb{T}}\left(\sum_{j=1}^{n} z_{j} x_{j}\right)^{\otimes n} \frac{d z_{1}}{z_{1}} \cdots \frac{d z_{n}}{z_{n}} \\
& =\sum_{j_{1}, \ldots, j_{n}=1}^{n}\left(\int_{z_{1}, \ldots, z_{n} \in \mathbb{T}} \prod_{r=1}^{n} z_{j_{r}} \frac{d z_{1}}{z_{1}} \cdots \frac{d z_{n}}{z_{n}}\right)\left(x_{j_{1}} \otimes \cdots \otimes x_{j_{n}}\right) \\
& =\sum_{j_{1}, \ldots, j_{n}=1}^{n}\left(\prod_{s=1}^{n} \int_{z \in \mathbb{T}} z^{\left|\left\{r: j_{r}=s\right\}\right|} \frac{d z}{z}\right)\left(x_{j_{1}} \otimes \cdots \otimes x_{j_{n}}\right) \\
& =(2 \pi i)^{n} \sum_{\pi \in S_{n}} x_{\pi(1)} \otimes \cdots \otimes x_{\pi(n)},
\end{aligned}
$$

where $\mathbb{T}$ denotes the unit circle in the complex plane. It follows that $\Phi_{n}\left(x_{1} \otimes \cdots \otimes x_{n}\right)$ belongs to $\mathcal{B}$ for any choice of $x_{1}, \ldots, x_{n}$, and hence $\mathcal{B}$ coincides with $\operatorname{Sym}^{n} \mathbb{M}_{d}$.

On the other hand, it is clear by definition that $\operatorname{Sym}^{n} \mathbb{M}_{d}$ contains the algebra $\left\{u_{n}(x): x \in \mathbb{M}_{d}\right\}^{\prime \prime}$ generated by the $u_{n}(x)$. Moreover, a consideration of the expansion for $u_{n}(x)^{n}$ shows that $x^{\otimes n}$ belongs to this double commutant for all $x \in \mathbb{M}_{d}$. For the sake of brevity let us observe this fact for $n=2$ and $n=3$ :

$$
\begin{aligned}
x \otimes x & =\frac{1}{2 !}\left(u_{2}(x)^{2}-u_{2}\left(x^{2}\right)\right) \\
x \otimes x \otimes x & =\frac{1}{3 !}\left(u_{3}(x)^{3}-3 u_{3}\left(x^{2}\right) u_{3}(x)-2 u_{3}\left(x^{3}\right)\right) .
\end{aligned}
$$

In fact, for all $x \in \mathbb{M}_{d}$, the tensor product $x^{\otimes n}$ belongs to the algebra $\operatorname{Alg}\left\{u_{n}\left(x^{p}\right): 1 \leq p \leq n\right\}$. Thus the second characterization of $\operatorname{Sym}^{n} \mathbb{M}_{d}$ follows.

Observe that as a consequence of this proof, we also have $\operatorname{Sym}^{n} \mathbb{M}_{d}=$ $\left\{u_{n}(x): x \in \mathbb{M}_{d, s a}\right\}^{\prime \prime}$. We can now explicitly link the noise commutant for these channels with representation theory of the symmetric group.

Theorem 4.3. Let $\mathcal{S} \subset \mathbb{M}_{d, s a}$ be a maximal system, then

$$
\operatorname{Fix}\left(\mathcal{E}_{\mathcal{S}}\right)=\mathcal{A}_{\mathcal{S}}^{\prime}=\pi\left(S_{n}\right)^{\prime \prime}
$$


Moreover, for an arbitrary finite set $\mathcal{S} \subset \mathbb{M}_{d, s a}$, we have

$$
\operatorname{Fix}\left(\mathcal{E}_{\mathcal{S}}\right) \supseteq \pi\left(S_{n}\right)^{\prime \prime}
$$

Proof. If $\mathcal{S}$ is maximal, then the interaction algebra $\operatorname{Fix}\left(\mathcal{E}_{\mathcal{S}}\right)^{\prime}=\mathcal{A}_{\mathcal{S}}=$ $\left\{u_{n}(x): x \in \mathcal{S}\right\}^{\prime \prime}=\left\{u_{n}(x): x \in \mathbb{M}_{d}\right\}^{\prime \prime}$ coincides with $\pi\left(S_{n}\right)^{\prime}$ by Lemma 4.2. For the second assertion, a given finite subset $\mathcal{S} \subset \mathbb{M}_{d, s a}$ is contained inside a maximal system $\mathcal{S}_{\max }$. Hence $\mathcal{A}_{\mathcal{S}} \subseteq \mathcal{A}_{\mathcal{S}_{\max }}$ and

$$
\operatorname{Fix}\left(\mathcal{E}_{\mathcal{S}}\right)=\mathcal{A}_{\mathcal{S}}^{\prime} \supseteq \mathcal{A}_{\mathcal{S}_{\max }}^{\prime}=\pi\left(S_{n}\right)^{\prime \prime} .
$$

\section{Computing Noiseless Subsystems Via Young Tableaux}

In this section, we collect well-known facts from the representation theory of $S_{n}$ that allow us to describe $\operatorname{Fix}\left(\mathcal{E}_{\mathcal{S}}\right)=\pi\left(S_{n}\right)^{\prime \prime}$ in an explicit manner. Recall that this is imperative for using the structure of the noise commutant to produce noiseless subsystems.

For the discussion in this section, we shall fix positive integers $d \geq 2$ and $n \geq 2$. Let $\{|0\rangle, \ldots,|d-1\rangle\}$ be the orthonormal basis for $\mathcal{H}_{d}$ corresponding to a given $d$-level quantum system, and let

$$
\left\{\left|i_{1} \cdots i_{n}\right\rangle: 0 \leq i_{j}<d, 1 \leq j \leq n\right\}
$$

be the corresponding basis for $\mathcal{H}_{d^{n}}$. Observe that the set of $n$-tuples $\left\{i_{1}, \ldots, i_{n}\right\}$ is in one-to-one correspondence with the set of functions $f:\{1, \ldots, n\} \rightarrow\{0, \ldots, d-1\}$. So we may define functions $k_{l}$ for $0 \leq l<d$ by

$$
k_{l}\left(i_{1}, \ldots, i_{n}\right)=\#\left\{1 \leq j \leq n \mid i_{j}=l\right\} \text { for } 0 \leq i_{j}<d,
$$

and we have $\sum_{l=0}^{d-1} k_{l}\left(i_{1}, \ldots, i_{n}\right)=n$.

Now, given positive integers $k_{0}, \ldots, k_{d-1}$ with each $0 \leq k_{l} \leq n$, we define a corresponding subspace of $\mathcal{H}_{d^{n}}$ by

$$
\mathcal{H}_{k_{0}, \ldots, k_{d-1}}=\operatorname{span}\left\{\left|i_{1} \cdots i_{n}\right\rangle: k_{l}\left(i_{1}, \ldots, i_{n}\right)=k_{l}, 0 \leq l<d\right\} .
$$


Notice that $\mathcal{H}_{d^{n}}=\bigoplus \mathcal{H}_{k_{0}, \ldots, k_{d-1}}$, where the direct sum runs over all choices of $k_{0}, \ldots, k_{d-1}$. Clearly, $\mathcal{H}_{k_{0}, \ldots, k_{d-1}}$ is an invariant (hence reducing) subspace for the action of the symmetric group $S_{n}$. More importantly, the irreducible subspaces, or equivalently the decomposition factors of $\mathcal{H}_{k_{0}, \ldots, k_{d-1}}$ are completely characterized. The key ingredient in this characterization is the notion of Young tableaux.

Given $\lambda_{1} \geq \lambda_{2} \geq \ldots \geq \lambda_{r}$, a non-increasing sequence of positive integers with $\sum_{i} \lambda_{i}=n$, put $\lambda=\left(\lambda_{1}, \ldots, \lambda_{r}\right)$. Then the associated $\lambda$-diagram is defined as

$$
[\lambda]=\left\{c_{i j}: 1 \leq i \leq r, 1 \leq j \leq \lambda_{i}\right\},
$$

where $c_{i j}$ denotes a 'cell' in $[\lambda]$. Simply put, $[\lambda]$ is a diagram with $d$ rows of cells which are left justified and $\lambda_{i}$ cells in the $i$ th row. A $\lambda$ tableau is a bijective function $t:[\lambda] \rightarrow\{1, \ldots, n\}$. Clearly, $S_{n}$ acts by composition $\sigma t=\sigma \circ t$ on $\lambda$-tableaux. Given a $\lambda$-tableau, the column stabilizer $C_{t}$ is the subgroup of $S_{n}$ which leaves the columns of $\lambda$ setwise fixed. Similarly, the row stabilizer $R_{t}$ is the subgroup of $S_{n}$ which leaves the rows of $\lambda$ setwise fixed. Two tableaux $t_{1}$ and $t_{2}$ are equivalent if there exists a permutation $\sigma \in R_{t_{1}}$ such that $\sigma t_{1}=t_{2}$. In particular, this means that the set of tabloids $\operatorname{Tab}_{\lambda}=\{\{t\}: t$ a $\lambda$-tableau $\}$ of equivalence classes is indexed by all partitions $\left(A_{1}, \ldots, A_{r}\right)$ of $\{1, \ldots, n\}$ such that the cardinalities $\left|A_{1}\right|=\lambda_{1}, \ldots,\left|A_{r}\right|=\lambda_{r}$. Given a $\lambda$-diagram $[\lambda]$, consider the $(i, j)$-cell $c_{i j}$ in $[\lambda]$. The hook length $h(i, j)$ for $c_{i j}$ is the number of cells directly below $c_{i j}$ in the $j$ th column of $[\lambda]$ plus the number of cells to the right of $c_{i j}$ in the $i$ th row of $[\lambda]$ plus one (for the cell $c_{i j}$ itself). Formally,

$$
h(i, j)=\lambda_{i}+\lambda_{j}^{\prime}+1-i-j,
$$

where $\lambda_{j}^{\prime}$ is the number of elements in the $j$-th column. Also recall that a tableau $t:[\lambda] \rightarrow\{1, \ldots, n\}$ is standard if the numbers increase along rows and increase down columns. The abstract $S_{n}$-module that has an 
orthonormal basis in bijective correspondence with elements of $\mathrm{Tab}_{\lambda}$ is denoted by $M^{\lambda}$, so that

$$
M^{\lambda}=\operatorname{span}\left\{e_{\{t\}}:\{t\} \in \operatorname{Tab}_{\lambda}\right\}
$$

The Specht module $\mathcal{S}^{\lambda}$ is the submodule of $M^{\lambda}$ generated by the 'polytabloids'

$$
e_{t} \equiv \sum_{\sigma \in C_{t}}(\operatorname{sgn} \sigma) e_{\sigma\{t\}} \in M^{\lambda}
$$

Let us summarize the following facts (see chapter 7 in [18]).

Theorem 5.1. Let $k_{0}, \ldots, k_{d-1}$ be positive integers and consider a partition of $\{1, \ldots, n\}$ into sets $A_{0}, \ldots, A_{d-1}$ with $\left|A_{l}\right|=k_{l}$. Let $\lambda$ be the non-increasing rearrangement of $\left(k_{0}, \ldots, k_{d-1}\right)$. Then $\mathcal{H}_{k_{0}, \ldots, k_{d-1}}$ is isomorphic as an $S_{n}$-module to $M^{\lambda}$.

Every polytabloid $e_{t}$ is a cyclic vector for the irreducible module $\mathcal{S}^{\lambda}$. The dimension of $\mathcal{S}^{\lambda}$ is given by the 'hook length formula'

$$
\operatorname{dim} \mathcal{S}^{\lambda}=\frac{n !}{\prod \text { hook lengths in }[\lambda]},
$$

and a basis for $\mathcal{S}^{\lambda}$ is given by

$$
\left\{e_{t}: t \text { standard } \lambda \text {-tableau }\right\} \text {. }
$$

Finally, every finite dimensional irreducible representation of $S_{n}$ is unitarily equivalent to a Specht module representation $\pi_{\lambda}$, where $\pi_{\lambda}$ is the representation of $S_{n}$ on $\mathcal{S}^{\lambda}$ defined by $\pi_{\lambda}(\sigma) e_{t} \equiv e_{\sigma t}$.

For the next discussion let us fix numbers $\left(k_{0}, \ldots, k_{d-1}\right)$ and let us denote by $\mu=\left(\mu_{0}, \ldots, \mu_{d-1}\right)$ the non-increasing rearrangement of $\left(k_{0}, \ldots, k_{d-1}\right)$. The $S_{n}$-module $\mathcal{H}_{k_{0}, \ldots, k_{d-1}}$ decomposes into a direct sum of irreducible submodules. Fortunately, these submodules and their multiplicity are completely characterized by Young's rule. Moreover, below we shall describe how the decomposition into irreducible submodules of $\mathcal{H}_{k_{0}, \ldots, k_{d-1}}$ is related to, and determined by, the decomposition of $\mathcal{H}_{\mu} \equiv \mathcal{H}_{\mu_{0}, \ldots, \mu_{d-1}}$. (This allows us to explicitly identify links 
between irreducible subspaces for the representation $\pi$.) Here the key combinatorial tool is the notion of a semistandard tableau.

We generalize the notion of $\lambda$-tableau, by saying that $T:[\lambda] \rightarrow \mathbb{N}$ is a $\lambda$-tableau of type $\mu=\left(\mu_{0}, \ldots ., \mu_{d-1}\right)$ if

$$
\#\left\{c_{i j}: T\left(c_{i j}\right)=l\right\}=\mu_{l} \quad \text { for } \quad l=0, \ldots, d-1 .
$$

Then $T$ is called semistandard if the numbers that $T$ assigns to the cells of the diagram determined by $\lambda$ are non-decreasing along rows and strictly increasing down columns. Let us fix a bijection $t_{0}:[\lambda] \rightarrow$ $\{1, \ldots, n\}$. Then $S_{n}$ acts on the sets $I(\lambda, \mu)$, the set of $\lambda$-tableau of type $\mu$, via

$$
\sigma(T)=T t_{0}^{-1} \sigma t_{0} \quad \text { for } \quad \sigma \in S_{n} .
$$

Given $t_{0}$, we will say that $T_{1}$ and $T_{2}$ are row (column) equivalent, and write $T_{1} \sim_{t_{0}}^{r} T_{2}$, if $\sigma T_{1}=\sigma T_{2}$ holds for all permutations $\sigma$ in the row (respectively column) stabilizer of $t_{0}$. In particular, this means that $T_{1}$ and $T_{2}$ are row equivalent if and only if $T_{2}$ is obtained from $T_{1}$ by permuting the entries in each row accordingly.

In order to define the linking module maps we first need an appropriate bijection. We denote by $\mathcal{P}_{\mu_{0}, \ldots, \mu_{d-1}}$ the set of partitions $\left(A_{0}, \ldots, A_{d-1}\right)$ of $\{1, \ldots, n\}$ such that $\left|A_{l}\right|=\mu_{l}$. Then $\mathcal{P}_{\mu_{0}, \ldots, \mu_{d-1}}$ induces a natural relabelling of the standard basis for $\mathcal{H}_{\mu}$ by

$$
f_{A_{0}, \ldots, A_{d-1}}=\left|i_{1} \cdots i_{n}\right\rangle
$$

where $A_{l}=\left\{1 \leq j \leq n \mid i_{j}=l\right\}$ for $0 \leq l<d$. (Every $n$-tuple $\left(i_{1}, \ldots, i_{n}\right)$ is associated with a unique $d$-tuple of sets $\left(A_{0}, \ldots, A_{d-1}\right)$ defined in this way.)

Next we define $\gamma_{t_{0}}: I(\lambda, \mu) \rightarrow \mathcal{P}_{\mu_{0}, \ldots, \mu_{d-1}}$ by

$$
\gamma_{t_{0}}(T)=\left(A_{0}, \ldots, A_{d-1}\right)
$$

where

$$
A_{l}=\left\{1 \leq j \leq n \mid T t_{0}^{-1}(j)=l\right\} \quad \text { for } \quad 0 \leq l<d
$$


Every $\lambda$-tableau $T$ of type $\mu$ induces an $S_{n}$-module map $\Theta_{T}: M^{\lambda} \rightarrow M^{\mu}$ by

$$
\Theta_{T}\left(e_{\left\{t_{0}\right\}}\right)=\sum_{T^{\prime} \sim_{t_{0}}^{r} T, \gamma_{t_{0}}\left(T^{\prime}\right)=\left(A_{0}, \ldots, A_{d-1}\right)} f_{A_{0}, \ldots, A_{d-1}} .
$$

Clearly this extends to an $S_{n}$-module homomorphism by defining

$$
\Theta_{T}\left(e_{\left\{\sigma\left(t_{0}\right)\right\}}\right)=\sigma\left(\Theta_{T}\left(e_{\left\{t_{0}\right\}}\right)\right) .
$$

This rather abstract description is in fact very concrete. Given indices $i_{1}, \ldots, i_{n} \in\{0, \ldots, d-1\}$ and a $\lambda$-tableau $t:[\lambda] \rightarrow\{1, \ldots, n\}$ we form the generalized tableau $t_{\left|i_{1} \cdots i_{n}\right\rangle}:[\lambda] \rightarrow\{0, \ldots, d-1\}$ by

$$
t_{\left|i_{1} \cdots i_{n}\right\rangle}\left(c_{i j}\right)=i_{t_{0}\left(c_{i j}\right)} .
$$

This means we write the entries $i_{1}, \ldots, i_{n}$ into $\lambda$ following the order given by $t_{0}$. Then we say that

$$
\left(i_{1}, \ldots, i_{n}\right) \sim_{t_{0}}\left(i_{1}^{\prime}, \ldots, i_{n}^{\prime}\right)
$$

if there exists a permutation $\sigma \in S_{n}$ such that $i_{j}^{\prime}=i_{\sigma(j)}$ for $1 \leq j \leq n$ and $t_{0}^{-1} \sigma t_{0}$ leaves the rows of $\lambda$ invariant. Therefore, we obtain

$$
\Theta_{T}\left(e_{\left\{t_{0}\right\}}\right)=\sum_{\left(i_{1}, \ldots, i_{n}\right) \sim_{t_{0}} \gamma_{t_{0}}(T)}\left|i_{1} \cdots i_{n}\right\rangle,
$$

where here we identify $\gamma_{t_{0}}(T)$ with the $n$-tuple determined by the partition $\gamma_{t_{0}}(T)=\left(A_{0}, \ldots, A_{d-1}\right)$ as in (3).

For example, let $d=3, n=5$ and let $t_{0}:[\lambda] \rightarrow\{1, \ldots, 5\}$ be given by

$$
t_{0}=\begin{array}{|l|l|l|l|}
\hline 1 & 3 & 2 & 4 \\
\hline 5 & \multicolumn{3}{|l}{} \\
\hline
\end{array}
$$

and $T:[\lambda] \rightarrow\{0,1,2\}$ be given by

$$
T=\begin{array}{|l|l|l|l|}
\hline 0 & 0 & 1 & 1 \\
\hline 2 & \multicolumn{4}{|l}{} \\
\hline
\end{array} .
$$

This yields, by reading off the entries from the corresponding position in the diagram,

$$
\gamma_{t_{0}}(T)=\left(A_{0}, A_{1}, A_{2}\right)=(\{1,3\},\{2,4\},\{5\}) .
$$


Following (3), $\gamma_{t_{0}}(T)$ is identified with $\left(i_{1}, i_{2}, i_{3}, i_{4}, i_{5}\right)=(0,1,0,1,2)$. Moreover, the list of equivalent indices is:

$$
\begin{array}{r}
\{(0,1,0,1,2),(0,1,1,0,2),(0,0,1,1,2),(1,0,0,1,2) \\
(1,0,1,0,2),(1,1,0,0,2)\} .
\end{array}
$$

Indeed, according to $t_{0}$ we have to fix the 5 th coordinate and the other four vary in all possible ways. Thus we have

$$
\Theta_{T}\left(e_{\{t\}}\right)=\sum_{\left(i_{1}, \ldots, i_{n}\right) \sim_{t} \gamma_{t}(T)}\left|i_{1} \cdots i_{n}\right\rangle \quad \text { for } \quad e_{\{t\}} \in M^{\lambda} .
$$

Following Young's rule (see chapter 2, [18]) we obtain:

Theorem 5.2. Let $\mu=\left(k_{0}^{*}, \ldots, k_{d-1}^{*}\right)$ be the non-increasing rearrangement of $\left(k_{0}, \ldots, k_{d-1}\right)$. Let $\lambda=\left(\lambda_{1}, \ldots, \lambda_{r}\right)$ be such that $\lambda_{1} \geq \ldots \geq \lambda_{r}$ and $\sum_{i} \lambda_{i}=n$. Then

$\mathcal{H}_{k_{0}, \ldots, k_{d-1}}^{\lambda} \equiv \operatorname{span}\left\{\Theta_{T}\left(\sum_{\sigma \in C_{t}}(\operatorname{sgn} \sigma) e_{\sigma\{t\}}\right): T \in I(\lambda, \mu), t \lambda\right.$-tableau $\}$

is an irreducible $S_{n}$-submodule. The restriction of the representation $\pi$ to $\mathcal{H}_{k_{0}, \ldots, k_{d-1}}^{\lambda}$ is equivalent to the irreducible representation $\pi_{\mu}$ of $S_{n}$ on $S^{\mu}$ and has multiplicity

$$
m=\#\{T: T \text { semistandard } \lambda \text {-tableau of type } \mu\} .
$$

If we collect all this information for all $\left(k_{0}, \ldots, k_{d-1}\right)$, we can describe the full representation $\pi$ of $\mathbb{C}\left[S_{n}\right]$ :

Corollary 5.3. Let $\lambda=\left(\lambda_{1}, \ldots, \lambda_{r}\right)$ be such that $\lambda_{1} \geq \ldots \geq \lambda_{r}$ and $\sum_{i} \lambda_{i}=n$, and let $P_{\lambda}$ be the projection of $\mathcal{H}_{d^{n}}$ onto

$$
\mathcal{H}^{\lambda} \equiv \bigoplus_{k_{0}, \ldots, k_{d-1}} \mathcal{H}_{k_{0}, \ldots, k_{d-1}}^{\lambda}
$$

where the sum indexes over all $k_{0}, \ldots, k_{d-1}$ such that $\sum_{l=0}^{d-1} k_{l}=n$. 
Then $P_{\lambda}$ is the minimal central projection for $\pi\left(S_{n}\right)^{\prime \prime}$ which supports the irreducible submodule $\mathcal{S}^{\lambda}$. Moreover, $P_{\lambda} \pi P_{\lambda}$ is equivalent to the representation $\pi_{\lambda}$ on $\mathcal{S}^{\lambda}$ with multiplicity

$$
m_{\lambda, d}=\sum_{\mu_{0} \geq \cdots \geq \mu_{d-1}} \operatorname{Arr}(\mu) \operatorname{SST}(\mu)
$$

where

$$
\operatorname{Arr}(\mu)=\#\left\{\left(k_{0}, \ldots, k_{d-1}\right):\left(k_{0}^{*}, \ldots, k_{d-1}^{*}\right)=\left(\mu_{0}, \ldots, \mu_{d-1}\right)\right\}
$$

and

$$
\begin{aligned}
& \operatorname{SST}(\mu)=\#\{T:[\lambda] \rightarrow\{0, \ldots, d-1\} \quad T \text { semistandard } \\
& \lambda \text {-tableau of type } \mu\} \text {. }
\end{aligned}
$$

In particular, for a maximal system $\mathcal{S}$,

$$
\operatorname{Fix}\left(\mathcal{E}_{S}\right)=\mathcal{A}_{\mathcal{S}}^{\prime}=\pi\left(S_{n}\right)^{\prime \prime} \cong \sum_{m_{\lambda, d} \neq 0} \mathbb{M}_{\operatorname{dim}\left(\mathcal{S}^{\lambda}\right)} \otimes \mathbb{1}_{m_{\lambda, d}}
$$

describes the representation in irreducible parts with multiplicity.

Corollary 5.4. Let $\mathcal{S}$ be a maximal system, then $\mathcal{A}_{\mathcal{S}}$ is isomorphic to

$$
\mathcal{A}_{\mathcal{S}} \cong \sum_{m_{\lambda, d} \neq 0} \mathbb{M}_{m_{\lambda, d}} \otimes \mathbb{1}_{\operatorname{dim} \mathcal{S}^{\lambda}}
$$

and the multiplicity of the component $\mathbb{M}_{m_{\lambda, d}}$ is given by $\operatorname{dim} \mathcal{S}^{\lambda}$.

Let us mention that $\mathcal{H}^{\lambda}$ may also be described by the so-called Garnier relations. Given $\lambda=\left(\lambda_{1}, \ldots, \lambda_{r}\right)$, we fix the tableau $T_{\lambda}$ such that $T_{\lambda}\left(c_{k j}\right)=k$ for all cells $c_{k j}$ in $[\lambda]$. It follows that every index $i=\left(i_{1}, \ldots, i_{n}\right)$ defines a tableau $T_{i}:[\lambda] \rightarrow\{0, \ldots, d-1\}$ given by $T_{i}\left(c_{k j}\right)=i_{T_{\lambda}\left(c_{k j}\right)}$. Let $G(J)$ be the collection of coset representatives $\{\nu X: \nu \in Y\}$, where $Y$ is the subgroup of $S_{n}$ which fixes every element outside both $C_{h}\left(T_{\lambda}\right) \cup J$ and $Y=X \cap C(T)$. Then as is proved in [15] (p.66, 5.2b), $|\psi\rangle \in \mathcal{H}^{\lambda}$ if and only if

(1) $\langle\psi \| i\rangle=0$ for all $i$ such that $T_{i}$ has equal entries in two distinct places in the same column. 
(2) $\pi(\sigma)(|\psi\rangle)=\operatorname{sgn}(\sigma)|\psi\rangle$ for all $\sigma$ in the column stabilizer of $T_{\lambda}$.

(3) $\sum_{\nu \in G(J)} \operatorname{sgn}(\nu) \pi\left(\nu^{-1}\right)|\psi\rangle=0$ for any non-empty set in the column stabilizer of $C_{h+1}\left(T_{\lambda}\right)$.

\section{Examples}

6.1. The case $d=2$ and general $n$. As mentioned above, the case of $d=2$ and general $n$ was extensively examined in [16]. Let us indicate how this can be accomplished with Young tableaux.

When $d=2$, we have the pairs $\left(k_{1}, k_{2}\right)$ given by $(n-k, k)$ where $k=0, \ldots, n$. In terms of $\lambda$-tableau we have to calculate $m_{\lambda, 2}$. In terms of types we only have to consider diagrams $\mu_{k}=(n-k, k)$ where $0 \leq k \leq 2 n$. But we have to be aware that every type allows combinations $(n-k, k)$ and $(k, n-k)$. Given $\lambda=\left(\lambda_{1}, \ldots, \lambda_{r}\right)$, we observe that to obtain a semistandard tableau, we must have $r=2$. Indeed, we are forced to put 0's in the first row on the first $\lambda_{2}$ positions and 1's in the second row. Thus for fixed $k, j$ with $2 k \leq n$ an $2 j \leq n$, we need $k \leq j$ in order to produce a $\lambda$-tableau of type $\mu$. Since, we also know that there are $n-k 0$ 's, we do not have a choice and we have to put them all in the first row one after another. Thus for a fixed $\lambda$, we find

$$
m_{(n-j, j), 2}=\sum_{k \leq j}^{\lfloor n / 2\rfloor} 2+1=2(\lfloor n / 2\rfloor-j)+1
$$

if $n$ is even and

$$
m_{(n-j, j), 2}=2(\lfloor n / 2\rfloor-j)
$$

if $n$ is odd, where $\lfloor\cdot\rfloor$ denotes the greatest integer part of some number.

We also have to understand $\operatorname{dim}\left(\mathcal{S}^{\lambda}\right)$. If $\lambda=0$, we get $\operatorname{dim}\left(\mathcal{S}^{(n, 0)}\right)=$ 1. If $1 \leq j<\frac{n}{2}$, we see for cells $c_{1 l}$ with $l \leq j$ the hook length is $1+(n-j+1-i)$. This yields $n(n-2 j+1) / j !(n-j+1) !$ and hence

$$
\operatorname{dim}\left(\mathcal{S}^{(n-j, j)}\right)=\left\{\begin{array}{ll}
1 & \text { if } j=0 \\
\frac{n-2 j+1}{n+1}\left(\begin{array}{c}
n+1 \\
j
\end{array}\right) & \text { if } 1<j \leq \frac{n}{2}
\end{array} .\right.
$$


Let us consider the examples $n=4$ and $n=5$. Then

$$
\operatorname{dim} \mathcal{S}^{(4,0)}=1, \quad \operatorname{dim} \mathcal{S}^{(3,1)}=3, \quad \operatorname{dim} \mathcal{S}^{(2,2)}=2
$$

and

$$
m_{(4,0), 2}=5, \quad m_{(3,1), 2}=3, \quad m_{(2,2), 2}=1 .
$$

In the case $n=5$, we have

$$
\operatorname{dim} \mathcal{S}^{(5,0)}=1, \quad \operatorname{dim} \mathcal{S}^{(4,1)}=4, \quad \operatorname{dim} \mathcal{S}^{(3,2)}=5
$$

and

$$
m_{(5,0), 2}=6, \quad m_{(4,1), 2}=4, \quad m_{(3,2), 2}=2 .
$$

Bases for $\mathcal{H}_{2^{n}}$ which yield the associated algebra decompositions may be computed as well. Below we do this for a more intricate example.

6.2. The case $d=3$ and $n=4$. If $d=3$ and $n=4$, then the set of $\lambda$-diagrams which admit semistandard tableaux is given by

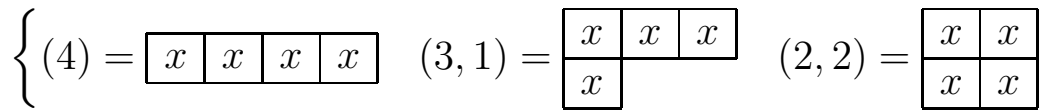

$$
\begin{aligned}
& \left.(2,1,1)=\begin{array}{|l|l|}
\hline x & x \\
\hline x & \\
\cline { 1 - 1 } x &
\end{array}\right\} .
\end{aligned}
$$

In this case, $\pi\left(S_{4}\right)$ acts on $\mathcal{H}_{d^{n}}=\mathcal{H}_{81}$. As in Theorem 5.1. $M^{(4)}$ is isomorphic to $\mathcal{H}_{4,0,0}, \mathcal{H}_{0,4,0}$ and $\mathcal{H}_{0,0,4} ; M^{(2,2)}$ is isomorphic to $\mathcal{H}_{2,2,0}$, $\mathcal{H}_{2,0,2}$ and $\mathcal{H}_{0,2,2} ; M^{(2,1,1)}$ is isomorphic to $\mathcal{H}_{2,1,1}, \mathcal{H}_{1,2,1}$ and $\mathcal{H}_{1,1,2}$; etc, so that the multiplicities for the $M^{\lambda}$ are 3 for $M^{(4)}, M^{(2,2)}$ and $M^{(2,1,1)}$ and 6 for $M^{(3,1)}$. The dimensions of the Specht modules $\mathcal{S}^{\lambda}$ using the hook length formula are given by

$$
\operatorname{dim} \mathcal{S}^{(4)}=1, \quad \operatorname{dim} \mathcal{S}^{(3,1)}=3, \quad \operatorname{dim} \mathcal{S}^{(2,2)}=2, \quad \operatorname{dim} \mathcal{S}^{(2,1,1)}=3
$$

Now, we have to compute the multiplicities of $\mathcal{S}^{\lambda}$ in $M^{\mu}$. If $\mu=(4)(=$ $(4,0,0))$, then the only semistandard tableau of type $\mu$ is $\lambda=(4)$ with 0 in each cell. Thus $M^{(4)} \cong \mathcal{S}^{(4)}$. Further, every $M^{\mu}$ supports a single 
copy of $\mathcal{S}^{(4)}$ via the $\lambda=(4)$-tableau with cell entries given by $\mu$. For $\mu=(3,1)$, the possible semistandard tableaux are

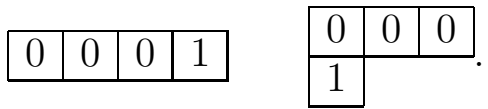

This gives

$$
M^{(3,1)} \cong \mathcal{S}^{(4)} \oplus \mathcal{S}^{(3,1)}
$$

For $\mu=(2,2)$, we have

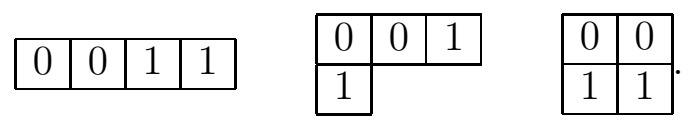

Thus we obtain

$$
M^{(2,2)} \cong \mathcal{S}^{(4)} \oplus \mathcal{S}^{(3,1)} \oplus \mathcal{S}^{(2,2)}
$$

Finally, for $\mu=(2,1,1)$ we find

$$
\begin{array}{|l|l|l|l|}
\hline 0 & 0 & 1 & 2 \\
\hline
\end{array}
$$
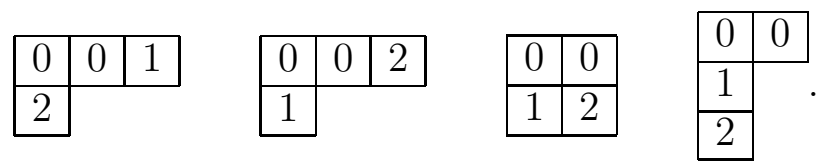

This means

$$
M^{(2,1,1)} \cong \mathcal{S}^{(4)} \oplus\left(\mathcal{S}^{(3,1)} \otimes \mathbb{1}_{2}\right) \oplus \mathcal{S}^{(2,2)} \oplus \mathcal{S}^{(2,1,1)} .
$$

Putting this all together, we find the module decomposition of $\pi\left(S_{4}\right)$ is given by

$$
\pi\left(S_{4}\right)=\left(M^{(4)} \otimes \mathbb{1}_{3}\right) \oplus\left(M^{(3,1)} \otimes \mathbb{1}_{6}\right) \oplus\left(M^{(2,2)} \otimes \mathbb{1}_{3}\right) \oplus\left(M^{(2,1,1)} \otimes \mathbb{1}_{3}\right)
$$

(4) $\quad=\left(\mathcal{S}^{(4)} \otimes \mathbb{1}_{3}\right) \oplus\left(\left(\mathcal{S}^{(4)} \oplus \mathcal{S}^{(3,1)}\right) \otimes \mathbb{1}_{6}\right)$

(5) $\oplus\left(\left(\mathcal{S}^{(4)} \oplus \mathcal{S}^{(3,1)} \oplus \mathcal{S}^{(2,2)}\right) \otimes \mathbb{1}_{3}\right)$

(6) $\oplus\left(\left(\mathcal{S}^{(4)} \oplus\left(\mathcal{S}^{(3,1)} \otimes \mathbb{1}_{2}\right) \oplus \mathcal{S}^{(2,2)} \oplus \mathcal{S}^{(2,1,1)}\right) \otimes \mathbb{1}_{3}\right)$

$(7) \quad=\left(\mathcal{S}^{(4)} \otimes \mathbb{1}_{15}\right) \oplus\left(\mathcal{S}^{(3,1)} \otimes \mathbb{1}_{15}\right) \oplus\left(\mathcal{S}^{(2,2)} \otimes \mathbb{1}_{6}\right) \oplus\left(\mathcal{S}^{(2,1,1)} \otimes \mathbb{1}_{3}\right)$.

The direct sums in (44), (5) and (6) are understood to be 'linked', as reflected in (7). It now follows that

$$
\begin{aligned}
\operatorname{Fix}\left(\mathcal{E}_{\mathcal{S}}\right) & =\pi\left(S_{4}\right)^{\prime \prime} \\
& \cong\left(\mathbb{C} \otimes \mathbb{1}_{15}\right) \oplus\left(\mathbb{M}_{3} \otimes \mathbb{1}_{15}\right) \oplus\left(\mathbb{M}_{2} \otimes \mathbb{1}_{6}\right) \oplus\left(\mathbb{M}_{3} \otimes \mathbb{1}_{3}\right)
\end{aligned}
$$


Notice also that $\mathbb{M}_{3}$ is the largest full matrix algebra which can be injected into $\operatorname{Fix}\left(\mathcal{E}_{\mathcal{S}}\right)$ as a subalgebra.

Let us now describe the bases for the decomposition

$$
\begin{aligned}
\mathcal{H}_{3^{4}}= & \left(\mathcal{H}_{4,0,0} \oplus \mathcal{H}_{0,4,0} \oplus \mathcal{H}_{0,0,4}\right) \oplus\left(\mathcal{H}_{2,1,1} \oplus \mathcal{H}_{1,2,1} \oplus \mathcal{H}_{1,1,2}\right) \\
& \oplus\left(\mathcal{H}_{3,1,0} \oplus \mathcal{H}_{3,0,1} \oplus \mathcal{H}_{0,3,1} \oplus \mathcal{H}_{1,3,0} \oplus \mathcal{H}_{0,1,3} \oplus \mathcal{H}_{1,0,3}\right)
\end{aligned}
$$

which yields this algebra decomposition. This is easy for $\lambda=(4)$. Indeed, for every $\mathcal{H}_{k_{0}, k_{1}, k_{2}}$ this is given by the invariant average vector

$$
h^{(4)}=\sum_{k_{0}\left(i_{1}, i_{2}, i_{3}, i_{4}\right)=k_{0}, \ldots, k_{2}\left(i_{1}, i_{2}, i_{3}, i_{4}\right)=k_{2}}\left|i_{1} i_{2} i_{3} i_{4}\right\rangle .
$$

In the following we will only discuss the case where $k_{0} \geq k_{1} \geq k_{2}$ (i.e., $\mathcal{H}_{4,0,0}, \mathcal{H}_{3,1,0}, \mathcal{H}_{2,1,1}$ ). For $\lambda=\mu$, we have a natural embedding $\mathcal{S}^{\lambda} \subseteq M^{\lambda} \cong \mathcal{H}_{k_{0}, k_{1}, k_{2}}$ given by

$$
h_{t}=\sum_{\sigma \in C_{t}} \operatorname{sgn}(\sigma) \sum_{\left(i_{1}, \ldots, i_{4}\right) \sim_{t}\left(i_{1}^{t}, \ldots, i_{4}^{t}\right)}\left|i_{1} i_{2} i_{3} i_{4}\right\rangle,
$$

for all $\lambda$-tableau $t$ of type $\mu$. Let us illustrate this in our examples. If $\lambda=(3,1)$, we have 3 standard tableaux

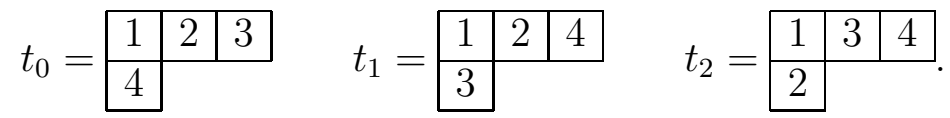

The column stabilizer of $t_{0}, t_{1}, t_{2}$ is $C_{t_{0}}=\{1,(14)\}, C_{t_{1}}=\{1,(13)\}$, $C_{t_{2}}=\{1,(12)\}$. The space $\mathcal{H}_{(3,1)}$ has the basis

$$
|0001\rangle, \quad|0010\rangle, \quad|0100\rangle, \quad|1000\rangle .
$$

Now, we define on $\mathcal{H}_{(3,1)}$

$$
A_{t_{i}}=\sum_{\sigma \in C_{t_{i}}} \operatorname{sgn}(\sigma) \pi(\sigma) .
$$

The range of $A_{t_{i}}$ is given by the vectors

$$
\begin{aligned}
& h_{t_{0}}=|0001\rangle-|1000\rangle, \\
& h_{t_{1}}=|0010\rangle-|1000\rangle, \\
& h_{t_{2}}=|0100\rangle-|1000\rangle .
\end{aligned}
$$


This provides us with the basis for

$$
M^{(3,1)} \cong \mathcal{H}_{(3,1)}=\operatorname{span}\left\{h^{(4)}\right\} \oplus \operatorname{span}\left\{h_{t_{0}}, h_{t_{1}}, h_{t_{2}}\right\}
$$

Now, we consider $\mathcal{H}_{(2,2,0)}$ spanned by

$$
|0011\rangle, \quad|0110\rangle, \quad|0101\rangle, \quad|1100\rangle, \quad|1010\rangle, \quad|1001\rangle \text {. }
$$

\begin{tabular}{|c|c|c|c|c|c|c|c|c|c|c|c|c|}
\hline 0 & \begin{tabular}{l|l}
0 & 1
\end{tabular} & 0 & 1 & 1 & 0 & \begin{tabular}{l|l}
1 & 0
\end{tabular} & 1 & \begin{tabular}{l|l}
1 & 0
\end{tabular} & 1 & \begin{tabular}{l|l}
0 & 1
\end{tabular} & 1 & \begin{tabular}{l|l}
0 & 0
\end{tabular} \\
\hline 1 & & 0 & & & 1 & & 0 & & \begin{tabular}{|l|}
0 \\
\end{tabular} & & 1 & \\
\hline
\end{tabular}

For $\lambda=(3,1)$ we have the following list of $\lambda$-tableaux of type $(2,2)$

Here we used $t_{0}=$\begin{tabular}{|l|l|l|}
\hline 1 & 2 & 3 \\
\hline 4 & & . Only the first tableaux is semistandard
\end{tabular} and yields an injection

$$
\Theta \equiv \Theta \begin{array}{|l|l|l}
\hline 0 & 0 & 1 \\
\hline 1 & &
\end{array} \mathcal{S}^{(3,1)} \rightarrow \mathcal{H}_{(2,2)}
$$

with

$$
\Theta\left(e_{\left\{t_{0}\right\}}\right)=\sum_{\left(i_{1}, \ldots, i_{4}\right) \sim_{t_{0}}(0,0,1,1)}\left|i_{1} i_{2} i_{3} i_{4}\right\rangle
$$

This means

$$
\Theta\left(e_{\left\{t_{0}\right\}}\right)=|0011\rangle+|0101\rangle+|1001\rangle .
$$

(See the example in the last section for $n=5$.) Further, this vector is a cyclic vector for the image of $\mathcal{S}^{(3,1)}$ in $\mathcal{H}_{(2,2)}$. The polytabloid is

$$
e_{t_{0}}=\sum_{\sigma \in C_{t_{0}}} \operatorname{sgn}(\sigma) \sigma e_{\left\{t_{0}\right\}}=e_{\left\{t_{0}\right\}}-(14) e_{\left\{t_{0}\right\}} .
$$

Therefore $\Theta\left(\mathcal{S}^{(3,1)}\right)$ is the module generated by

$$
\Theta\left(e_{t_{0}}\right)=h_{t_{0}}=|0011\rangle-|1010\rangle+|0101\rangle-|1100\rangle .
$$

Equivalently,

$$
\begin{aligned}
\Theta\left(\mathcal{S}^{(3,1)}\right)= & \operatorname{span}\left\{h_{t_{0}},(12) h_{t_{0}},(13) h_{t_{0}}\right\} \\
= & \operatorname{span}\{|0011\rangle-|1010\rangle+|0101\rangle-|1100\rangle, \\
& |1100\rangle-|1001\rangle+|0110\rangle-|0011\rangle, \\
& |0110\rangle-|0101\rangle+|1010\rangle-|1001\rangle\} .
\end{aligned}
$$


Another way to find a basis is to consider $t_{1}=$\begin{tabular}{|l|l|l|}
\hline 1 & 2 & 4 \\
\hline 3 &
\end{tabular} . In this case, $C_{t_{1}}=\{1,(13)\}$

$$
\Theta\left(e_{\left\{t_{1}\right\}}\right)=|0011\rangle+|0110\rangle+|1010\rangle
$$

and

$$
h_{t_{1}}=\Theta\left(e_{\left\{t_{1}\right\}}\right)-(13) \Theta\left(e_{\left\{t_{1}\right\}}\right)=|0011\rangle-|1001\rangle+|0110\rangle-|1100\rangle .
$$

Similarly for $t_{2}=$\begin{tabular}{|l|l|l}
\hline 1 & 3 & 4 \\
\hline 2 & &
\end{tabular} , we have $C_{t_{1}}=\{1,(12)\}$ and

$$
\Theta\left(e_{\left\{t_{2}\right\}}\right)=|0101\rangle+|0110\rangle+|1100\rangle
$$

and

$$
h_{t_{2}}=\Theta\left(e_{\left\{t_{2}\right\}}\right)-(12) \Theta\left(e_{\left\{t_{2}\right\}}\right)=|0101\rangle-|1001\rangle+|0110\rangle-|1010\rangle \text {. }
$$

The copy of $\mathcal{S}^{(2,2)}$ in $\mathcal{H}_{(2,2)}$ is again easy to find. We recall that $\mathcal{S}^{(2,2)}$ is spanned by the standard tableaux $\left\{e_{s_{0}}, e_{s_{1}}\right\}$ where

$$
s_{0}=\begin{array}{|l|l|}
\hline 1 & 2 \\
\hline 3 & 4 \\
\hline
\end{array} \quad s_{1}=\begin{array}{|l|l|}
\hline 1 & 3 \\
\hline 2 & 4 \\
\hline
\end{array}
$$

The column stabilizers are given by $C_{s_{0}}=\{1,(13),(24),(13)(24)\}$ and by $C_{s_{1}}=\{1,(12),(34),(12)(34)\}$. This yields operators on $\mathcal{H}_{(2,2)}$,

$$
A_{s_{0}}=1-\pi((13))-\pi((24))+\pi((13)(24))
$$

and

$$
A_{s_{1}}=1-\pi((12))-\pi((34))+\pi((12)(34)) .
$$

Applied to the unit vectors, we find the ranges

$$
\operatorname{Ran}\left(A_{s_{0}}\right)=|0110\rangle-|1010\rangle-|0101\rangle+|1001\rangle
$$

and

$$
\operatorname{Ran}\left(A_{s_{1}}\right)=|0011\rangle-|1001\rangle-|0110\rangle+|1100\rangle .
$$

Finally, we consider $\mathcal{H}_{(2,1,1)}$ with basis

$$
\begin{array}{r}
\{|0012\rangle,|0021\rangle,|0102\rangle,|0120\rangle,|0201\rangle,|0210\rangle,|1002\rangle,|1020\rangle,|1200\rangle \\
|2001\rangle,|2010\rangle,|2100\rangle\} .
\end{array}
$$


The representation of $\mathcal{S}^{(4)}$ is 1-dimensional, given by the average of all these vectors. There are two copies of $\mathcal{S}^{(3,1)}$ corresponding to the two $(3,1)$-tableaux of type $(2,1,1)$

$$
T=\begin{array}{|l|l|l|}
\hline 0 & 0 & 1
\end{array} \quad T^{\prime}=\begin{array}{|l|l|l|}
\hline 0 & 0 & 2 \\
\hline 2 & & \multicolumn{1}{|l}{} \\
\hline
\end{array}
$$

The basis for $\mathcal{S}^{(3,1)}$ is given by $\left\{e_{t_{0}}, e_{t_{1}}, e_{t_{2}}\right\}$ where

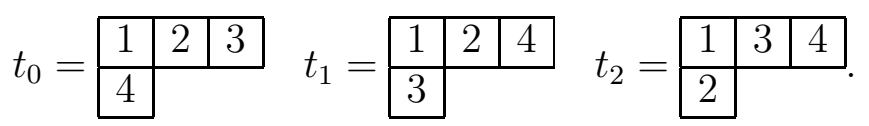

Following the definition of $\Theta_{T}\left(e_{t_{j}}\right)$, we get

$$
\gamma_{t_{0}}(T)=(0,0,1,2), \quad \gamma_{t_{1}}(T)=(0,0,2,1), \quad \gamma_{t_{2}}(T)=(0,2,0,1)
$$

Using row equivalence, we are allowed to permute the entries $\{1,2,3\}$ for $t_{0}$, the entries $\{1,2,4\}$ for $t_{1}$ and $\{1,3,4\}$ for $t_{2}$ and thus

$$
\begin{aligned}
& \Theta_{T}\left(e_{\left\{t_{0}\right\}}\right)=|0012\rangle+|0102\rangle+|1002\rangle, \\
& \Theta_{T}\left(e_{\left\{t_{1}\right\}}\right)=|0021\rangle+|0120\rangle+|1020\rangle, \\
& \Theta_{T}\left(e_{\left\{t_{2}\right\}}\right)=|0201\rangle+|0210\rangle+|1200\rangle .
\end{aligned}
$$

For $t_{0}, t_{1}, t_{2}$ we have to apply, respectively, $A_{T, t_{0}}=\mathbb{1}-\pi(14), A_{T, t_{1}}=$ $\mathbb{1}-\pi(13)$ and $A_{T, t_{2}}=\mathbb{1}-\pi(12)$ in order to obtain the image of the polytabloids:

$$
\begin{aligned}
& h_{T, t_{0}}=|0012\rangle-|2010\rangle+|0102\rangle-|2100\rangle+|1002\rangle-|2001\rangle, \\
& h_{T, t_{1}}=|0021\rangle-|2001\rangle+|0120\rangle-|2100\rangle+|1020\rangle-|2010\rangle, \\
& h_{T, t_{2}}=|0201\rangle-|2001\rangle+|0210\rangle-|2010\rangle+|1200\rangle-|2100\rangle .
\end{aligned}
$$

This is our first copy of $\mathcal{S}^{(3,1)}$. For the second, we exercise the same procedure in the case of $T^{\prime}$.

$$
\begin{aligned}
& \Theta_{T^{\prime}}\left(e_{\left\{t_{0}\right\}}\right)=|0021\rangle+|0201\rangle+|2001\rangle, \\
& \Theta_{T^{\prime}}\left(e_{\left\{t_{1}\right\}}\right)=|0012\rangle+|0210\rangle+|2010\rangle, \\
& \Theta_{T^{\prime}}\left(e_{\left\{t_{2}\right\}}\right)=|0102\rangle+|0120\rangle+|2100\rangle .
\end{aligned}
$$


This provides us with

$$
\begin{aligned}
& h_{T^{\prime}, t_{0}}=|0021\rangle-|1020\rangle+|0201\rangle-|1200\rangle+|2001\rangle-|1002\rangle, \\
& h_{T^{\prime}, t_{1}}=|0012\rangle-|1002\rangle+|0210\rangle-|1200\rangle+|2010\rangle-|1020\rangle, \\
& h_{T^{\prime}, t_{2}}=|0102\rangle-|1002\rangle+|0120\rangle-|1020\rangle+|2100\rangle-|1200\rangle .
\end{aligned}
$$

We have one copy of $\mathcal{S}^{(2,2)}$ which is spanned by

$$
s_{0}=\begin{array}{|l|l|}
\hline 1 & 2 \\
\hline 3 & 4 \\
\hline
\end{array} \quad s_{1}=\begin{array}{|l|l|}
\hline 1 & 3 \\
\hline 2 & 4 \\
\hline
\end{array}
$$

Our $(2,2)$ tableau of type $(2,1,1)$ is given by $T=$\begin{tabular}{|l|l|}
\hline 0 & 0 \\
\hline 1 & 2 \\
\hline
\end{tabular} . This gives

$$
\Theta\left(e_{\left\{s_{0}\right\}}\right)=|0012\rangle+|0021\rangle \text { and } \Theta\left(e_{\left\{s_{1}\right\}}\right)=|0102\rangle+|0201\rangle \text {. }
$$

The operator is $\mathbb{1}-\pi(13)-\pi(24)+\pi((13)(24))$, determined by $C_{s_{0}}=$ $\{1,(13),(24),(13)(24)\}$, and thus

$$
h_{s_{0}}=|0012\rangle-|1002\rangle-|0210\rangle+|1200\rangle+|0021\rangle-|2001\rangle-|0120\rangle+|2100\rangle
$$

and similarly for $s_{1}$ we apply $\mathbb{1}-\pi(12)-\pi(34)+\pi((12)(34))$ to obtain

$$
h_{s_{1}}=|0102\rangle-|1002\rangle-|0120\rangle+|1020\rangle+|0201\rangle-|2001\rangle-|0210\rangle+|2010\rangle \text {. }
$$

Finally we consider the copy of $\mathcal{S}^{(2,1,1)}$, which has basis $\left\{e_{r_{0}}, e_{r_{1}}, e_{r_{2}}\right\}$ where

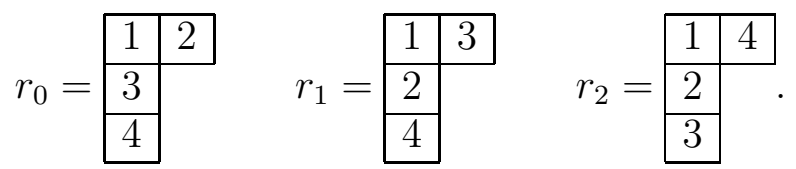

Here $T=$\begin{tabular}{|l|l|}
\hline 0 & 0 \\
\hline 1 & \\
\cline { 1 - 1 } 2 & . This yields \\
&
\end{tabular}

$$
\Theta_{T}\left(e_{\left\{r_{0}\right\}}\right)=|0012\rangle, \quad \Theta_{T}\left(e_{\left\{r_{1}\right\}}\right)=|0102\rangle, \quad \Theta_{T}\left(e_{\left\{r_{2}\right\}}\right)=|0120\rangle .
$$

The column stabilizer of $r_{2}$ is given by all permutations which leave $\{1,2,3\}$ invariant. This yields

$$
h_{r_{2}}=|0120\rangle-|0210\rangle-|1020\rangle+|1200\rangle+|2010\rangle-|2100\rangle \text {. }
$$


Similarly, we have to look for all permutations of $\{1,3,4\}$ in the column stabilizer of $r_{0}$ and we obtain

$$
h_{r_{0}}=|0012\rangle-|0021\rangle-|1002\rangle+|1020\rangle+|2001\rangle-|2010\rangle .
$$

For the column stabilizer of $r_{1}$, we may permute $\{1,2,4\}$ and hence

$$
h_{r_{1}}=|0102\rangle-|0201\rangle-|1002\rangle+|1200\rangle+|2001\rangle-|2100\rangle \text {. }
$$

By equation (8), the largest full matrix algebra $\mathbb{M}_{k}$ that can be injected into the noise commutant here is $\mathbb{M}_{3}$, identified with the subalgebras of $\mathcal{A}_{\mathcal{S}}^{\prime}$ unitarily equivalent to either $\mathbb{1}_{15} \otimes \mathbb{M}_{3}$ or $\mathbb{1}_{3} \otimes \mathbb{M}_{3}$. Let us explicitly identify the copy of $\mathbb{1}_{3} \otimes \mathbb{M}_{3}$. The set $\left\{h_{r_{0}}, h_{r_{1}}, h_{r_{2}}\right\}$ yields the copy of $S^{(2,1,1)}$ inside $\mathcal{H}^{(2,1,1)}$. A similar analysis yields the basis for the copy of $S^{(2,1,1)}$ inside $\mathcal{H}^{(1,2,1)}$. It is generated by $T^{\prime}=$\begin{tabular}{|l|l|}
\hline 0 & 1 \\
\hline 1 &
\end{tabular} and in this case

$$
\begin{aligned}
& \Theta_{T^{\prime}}\left(e_{\left\{r_{0}\right\}}\right)=|0112\rangle+|1012\rangle, \\
& \Theta_{T^{\prime}}\left(e_{\left\{r_{1}\right\}}\right)=|0112\rangle+|1102\rangle, \\
& \Theta_{T^{\prime}}\left(e_{\left\{r_{2}\right\}}\right)=|0121\rangle+|1120\rangle .
\end{aligned}
$$

Thus we have

$$
\left\{\begin{array}{l}
h_{r_{0}}^{\prime}=|0112\rangle-|0121\rangle-|1102\rangle+|1120\rangle+|2101\rangle-|2110\rangle \\
h_{r_{1}}^{\prime}=|0112\rangle-|0211\rangle-|1012\rangle+|1210\rangle+|2011\rangle-|2110\rangle \\
h_{r_{2}}^{\prime}=|0121\rangle-|0211\rangle-|1021\rangle+|1201\rangle+|2011\rangle-|2101\rangle
\end{array} .\right.
$$

Further, the basis for the copy of $S^{(2,1,1)}$ inside $\mathcal{H}^{(1,1,2)}$ is generated by $T^{\prime \prime}=$\begin{tabular}{|l|l|}
\hline 0 & 2 \\
\hline 1 &
\end{tabular} and in this case

$$
\begin{aligned}
& \Theta_{T^{\prime \prime}}\left(e_{\left\{r_{0}\right\}}\right)=|0212\rangle+|2012\rangle, \\
& \Theta_{T^{\prime \prime}}\left(e_{\left\{r_{1}\right\}}\right)=|0122\rangle+|2102\rangle, \\
& \Theta_{T^{\prime \prime}}\left(e_{\left\{r_{2}\right\}}\right)=|0122\rangle+|2120\rangle .
\end{aligned}
$$

Thus we have

$$
\left\{\begin{array}{l}
h_{r_{0}}^{\prime \prime}=|0212\rangle-|0221\rangle-|1202\rangle+|1220\rangle+|2201\rangle-|2210\rangle \\
h_{r_{1}}^{\prime \prime}=|0122\rangle-|0221\rangle-|1022\rangle+|1220\rangle+|2021\rangle-|2120\rangle \\
h_{r_{2}}^{\prime \prime}=|0122\rangle-|0212\rangle-|1022\rangle+|1202\rangle+|2012\rangle-|2102\rangle
\end{array} .\right.
$$


Let $P_{(2,1,1)}$ be the projection of $\mathcal{H}$ onto the span of $\left\{h_{r_{i}}, h_{r_{j}}^{\prime}, h_{r_{k}}^{\prime \prime}\right.$ : $0 \leq i, j, k \leq 2\}$. Then $P_{(2,1,1)}$ is a minimal central projection for $\mathcal{A}_{\mathcal{S}}^{\prime}$ and the 'compression subalgebra' $P_{(2,1,1)} \mathcal{A}_{\mathcal{S}}^{\prime} P_{(2,1,1)}=\mathcal{A}_{\mathcal{S}}^{\prime} P_{(2,1,1)} \subset \mathcal{A}_{\mathcal{S}}^{\prime}$ is unitarily equivalent to $\mathbb{1}_{3} \otimes \mathbb{M}_{3}$. In fact, with respect to the ordered basis

$$
\left\{h_{r_{0}}, h_{r_{1}}, h_{r_{2}}, h_{r_{0}}^{\prime}, h_{r_{1}}^{\prime}, h_{r_{2}}^{\prime}, h_{r_{0}}^{\prime \prime}, h_{r_{1}}^{\prime \prime}, h_{r_{2}}^{\prime \prime}\right\}
$$

for $P_{(2,1,1)} \mathcal{H}$, we have the matrix representations

$$
\mathcal{A}_{\mathcal{S}}^{\prime} P_{(2,1,1)}=\left\{\left(\begin{array}{ccc}
A & 0 & 0 \\
0 & A & 0 \\
0 & 0 & A
\end{array}\right): A \in \mathbb{M}_{3}\right\} .
$$

Note that the subspaces spanned by the sets $\left\{h_{r_{i}}\right\},\left\{h_{r_{i}}^{\prime}\right\}$ and $\left\{h_{r_{i}}^{\prime \prime}\right\}$ are perpendicular, but the vectors within each of these sets do not form an orthogonal basis for the corresponding subspace.

\section{Conclusion}

We have investigated the operator algebras of fixed points for a class of quantum channels we call universal collective rotation channels $\mathcal{E}_{\mathcal{S}}$. This class includes as a subclass the well-known class of collective rotation channels. We showed that such channels always have an abundance of noiseless subsystems and gave a method for explicitly computing them. In particular, the Young tableaux machine gives a clean approach for this process. In lower dimensional cases (e.g. when $d=2$ ), our approach is more technical when compared to others in the literature (for instance [16]). However, an important advantage of the Young tableaux approach for higher dimensional cases is that it is particularly amenable to computations.

An issue we have not pursued here concerns the channels generated by non-maximal sets $\mathcal{S}$. The $d^{n}$-dimensional representations of $J_{x}, J_{y}, J_{z}$ considered in [16] provide such an example, but we would expect there to be other interesting non-trivial examples of channels 
$\mathcal{E}_{\mathcal{S}}$ for non-maximal $\mathcal{S}$. We emphasize that even for non-maximal $\mathcal{S}$ there is an abundance of noiseless subsystems because $\operatorname{Fix}\left(\mathcal{E}_{\mathcal{S}}\right)$ contains $\pi\left(S_{n}\right)^{\prime \prime}$.

We also wonder what other representations of $S_{n}$ correspond to physically meaningful unital channels, beyond $\pi$ and its subrepresentations (which correspond to the compressions of ucr-channels). The recent preprint [3] of Bacon, et al, appears to present further insights into this topic, and also shows how the unitary base change from the standard basis to the basis given by the Young tableaux can be efficiently computed using quantum circuits.

Acknowledgements. We are grateful to the referee for helpful comments. We would like to thank John Holbrook, Raymond Laflamme and David Poulin for enlightening conversations. The first author was partially supported by NSF grant DMS 03-01116. The second and third authors were partially supported by NSERC grants. The third author also gratefully acknowledges support from the Institute for Quantum Computing and the Perimeter Institute for Theoretical Physics.

\section{REFERENCES}

[1] D. Aharonov, M. Ben-Or, Fault-tolerant quantum computation with constant error, In Proc. 29th. Ann. ACM Symp. on Theory of Computing, page 176, New York, 1998, ACM. arxiv.org/quant-ph/9906129, quant-ph/9611025

[2] W. Arveson, An invitation to $\mathrm{C}^{*}$-algebras, Graduate Texts in Mathematics, No. 39, Springer-Verlag, New York-Heidelberg, 1976.

[3] D. Bacon, I.L. Chuang, A.W. Harrow, Efficient quantum circuits for Schur and Clebsch-Gordon transforms, arxiv.org/quant-ph/0407082.

[4] S.D. Bartlett, T. Rudolph, R.W. Spekkens, Decoherence-full subsystems and the cryptographic power of a private shared reference frame, arXiv.org/quant$\mathrm{ph} / 0403161$.

[5] S.D. Bartlett, T. Rudolph, R.W. Spekkens, Classical and quantum communication without a shared reference frame, Phys. Rev. Lett. 91, 027901 (2003).

[6] J.-C. Boileau, D. Gottesman, R. Laflamme, D. Poulin, R.W. Spekkens, Robust polarization-based quantum key distribution over collective-noise channel, arXiv.org/quant-ph/0306199.

[7] P. Busch, J. Singh, Luders theorem for unsharp quantum effects, Phys. Lett. A, 249 (1998), 10-24. 
[8] M.D. Choi, Completely positive linear maps on complex matrices, Lin. Alg. Appl. 10 (1975), 285-290.

[9] C. Cohen-Tannoudji, B. Diu, F. Laloe, Quantum Mechanics, Volume One 83 Two, John Wiley \& Sons, Toronto, 1977.

[10] K.R. Davidson, C*-algebras by example, Fields Institute Monographs, 6, Amer. Math. Soc., Providence, 1996.

[11] L.-M. Duan, G.-C. Guo, Preserving coherence in quantum computation by pairing quantum bits, Phys. Rev. Lett. 79 (1997), 1953.

[12] S. De Filippo, Quantum computation using decoherence-free states of the physical operator algebra, Phys. Rev. A 62, 052307 (2000).

[13] E.M. Fortunato, L. Viola, M.A. Pravia, E. Knill, R. Laflamme, T.F. Havel, D.G. Cory, Exploring noiseless subsystems via nuclear magnetic resonance, Phys. Rev. A 67, 062303 (2003).

[14] W. Fulton, J. Harris, Representation theory, a first course, Springer-Verlag New York, 1991.

[15] J.A. Green, Polynomial representations of $G L_{n}$, Lecture Notes in Mathematics 830, Springer-Verlag, New York, 1980.

[16] J.A. Holbrook, D.W. Kribs, R. Laflamme, D. Poulin, Noiseless subsystems for collective rotation channels in quantum information theory, Integral Equations \& Operator Theory, to appear.

[17] J.A. Holbrook, D.W. Kribs, R. Laflamme, Noiseless subsystems and the structure of the commutant in quantum error correction, Quantum Information Processing 2 (2003), 381-419.

[18] G. James, A. Kerber, The representation theory of the symmetric group, Encyclopedia of Mathematics and its Applications, Addison-Wesley Publishing Company, Toronto, 1981.

[19] M. Junge, Noncommutative Poisson random measure, preprint, 2003.

[20] J. Kempe, D. Bacon, D.A. Lidar, K.B. Whaley, Theory of decoherence-free fault-tolerant universal quantum computation, Phys. Rev. A 63, 042307 (2001).

[21] E. Knill, R. Laflamme, A. Ashikhmin, H. Barnum, L. Viola, W.H. Zurek, Introduction to Quantum Error Correction, Los Alamos Science, November 27, 2002.

[22] E. Knill, R. Laflamme, L. Viola, Theory of quantum error correction for general noise, Phys. Rev. Lett. 84 (2000), 2525-2528.

[23] E. Knill, R. Laflamme, W. H. Zurek, Resilient quantum computation: error models and thresholds, Science 279 (1998), 342-345.

[24] E. Knill, R. Laflamme, A theory of quantum error-correcting codes, Phys. Rev. A 55 (1997), 900.

[25] K. Kraus, States, Effects and Operations: Fundamental Notions of Quantum Theory, Lecture Notes in Physics, vol. 190, Berlin: Springer-Verlag, 1983.

[26] K. Kraus, General state changes in quantum theory, Ann. Physics 64 (1971), 311-335.

[27] D.W. Kribs, A quantum information theory primer for operator theorists, preprint, 2004.

[28] D.W. Kribs, Quantum channels, wavelets, dilations, and representations of $\mathcal{O}_{n}$, Proc. Edin. Math. Soc., 46 (2003).

[29] R. Laflamme, private communication. 
[30] D. A. Lidar, I. L. Chuang, K. B. Whaley, Decoherence free subspaces for quantum computation, Phys. Rev. Lett. 81 (1998), 2594.

[31] M.A. Nielsen, I.L. Chuang, Quantum computation and quantum information, Cambridge University Press, 2000.

[32] V. Paulsen, Completely bounded maps and operator algebras, Cambridge University Press, Cambridge, United Kingdom, 2002.

[33] J. Preskill, Reliable quantum computers, Proc. R. Soc. Lond. A, 454 (1998), $385-410$.

[34] W.F. Stinespring, Positive functions on $\mathrm{C}^{*}$-algebras, Proc. Amer. Math. Soc. 6 (1955), 211-216.

[35] M. Takesaki, Theory of operator algebras I, Springer-Verlag, New YorkHeidelberg, 1979.

[36] L. Viola, E. Knill, R. Laflamme, Constructing qubits in physical systems, J. Phys. A 34, 7067 (2001).

[37] L. Viola, E.M. Fortunato, M.A. Pravia, E. Knill, R. Laflamme, D.G. Cory, Experimental realization of noiseless subsystems for quantum information processing, Science 293, 2059 (2001).

[38] P. Zanardi, S. Lloyd, Topological protection and quantum noiseless subsystems, Phys. Rev. Lett. 90, 067902 (2003).

[39] P. Zanardi, Stabilizing quantum information, Phys. Rev. A 63, 012301 (2001).

[40] P. Zanardi, M. Rasetti, Noiseless quantum codes, Phys. Rev. Lett. 79 (1997), 3306.

[41] V. Zarikian, Algorithms for operator algebra calculations, Lin. Alg. Appl., to appear.

${ }^{1}$ Department of Mathematics, University of Illinois at UrbanaChampaign, Urbana, Illinois, USA 61801-2975

${ }^{2}$ Department of Mathematics and Statistics, University of Guelph, Guelph, ON, CANADA N1G 2W1.

${ }^{3}$ Institute for Quantum Computing, University of Waterloo, WaTERLOO, ON, CANADA N2L 3G1.

${ }^{4}$ Perimeter Institute for Theoretical Physics, 35 King St. North, WATERloo, ON, CANADA N2J 2W9.

E-mail address: junge@math.uiuc.edu, pkim@uoguelph.ca, dkribs@uoguelph.ca 